\title{
Sprache und Identität: Einleitung
}

Dass jedes Volk seine Sprache hat, scheint aus dem Blickwinkel unserer Kultur zunächst selbstverständlich. Seit der Antike gilt Sprache als ein, ja zuweilen als das Definitionsmerkmal des Volkes: „Der Inbegriff von Menschen, welche dieselbe Sprache reden“. ${ }^{1}$ Der moderne Nationalismus ging noch weiter und hat in der Sprache den Ausdruck der innersten "Seele' eines Volkes gesehen; oft wurde davon ein politischer Auftrag abgeleitet. ${ }^{2}$ „Soweit die deutsche Zunge reicht“, so Ernst Moritz Arndt 1813, sollte „ganz Deutschland“" geeint werden. ${ }^{3}$ Dementspechend war und ist die Durchsetzung und einheitliche Lehre der Nationalsprache, oft genug auch die Unterdrückung anderer auf dem Territorium eines Nationalstaates gesprochener Sprachen ein zentrales Anliegen vieler europäischer Nationalbewegungen. ${ }^{4}$ Im Europa des 21. Jahrhunderts werden Baskisch, Katalanisch, Irisch, Walisisch, Korsisch als unverzichtbare Wesensmerkmale ,unterdrückter ${ }^{6}$ Nationen propagiert, selbst dort, wo kaum jemand sie als Muttersprache spricht. Auch wo nationale Einheitlichkeit nicht gewaltsam angestrebt wird, kann der Sprachenstreit zu politischer Lähmung führen, so bei den Bemühungen, Belgien schrittweise sprachlich aufzugliedern. Die Debatte um ein paar zweisprachige Ortstafeln mehr oder weniger wurde noch im 21. Jahrhundert in Kärnten von führenden Politikern populistisch angeheizt.

Nach zwei Jahrhunderten nationaler Sprachpolitik wird deutlich, dass der Traum von der sprachlich und ethnisch einheitlichen Nation nicht realisierbar ist. Nicht einmal die alten Nationen Westeuropas, England, Frankreich und Spanien, sind frei von Widerständen gegen die nationale Einheitssprache; im Gegenteil, diese verliert eher an Boden. Auch kleine Nationen wie die Schweiz, Belgien oder Finnland sind mehrsprachig, während Sprachen wie Deutsch, Französisch, Englisch oder Niederländisch in mehreren Nationen als Mehrheitssprache gesprochen werden. Sprache und nationale Identität sind also selbst mit großem politischem Aufwand und in langen Zeiträumen nicht zur Deckung zu bringen, so ließe sich die bisherige Erfahrung der europäischen Moderne zusammenfassen.

Freilich, die moderne Nation ist letztlich territorial und nicht notwendiger Weise ethnisch begründet. Die unüberbrückbare Differenz zwischen Sprache und nationaler Identität könnte auch daran liegen. Sollten wir daher die Identitätswirksamkeit der Sprache auf der Ebene der Ethnie suchen? ${ }^{5}$ Aus dem modernen Befund ist diese Frage gar nicht so leicht zu beantworten, denn ethnische Gruppen sind in Europa, aber auch anderswo vom Prozess der Nationsbildung erfasst und in ihrer Rolle verändert worden: gleichgültig, ob sie selbst zur Nation wurden und nach Vereinheitlichung eines unweigerlich vielsprachigen Territoriums strebten, oder ob sie als Minderheit in einem fremden Nationalstaat Halt an der Sprache suchten. Die Geschichte des Frühmittelalters hingegen gibt gute Vorausset-

\footnotetext{
${ }^{1}$ Jakob Grimm, Reden bei der frankfurter Germanisten-Versammlung, in: ders., Auswahl aus den kleineren Schriften (Berlin 1871) 331-347, hier 331. Siehe dazu den Beitrag von Daniela Fruscione in diesem Band.

2 Patrick J. Geary, The Myth of Nations (Princeton 2002) 15-40; Joep Leerssen, National Thought in Europe. A Cultural History (Amsterdam 2006) 255-267.

3 Peter Alter, Nationalismus (Frankufurt am Main 1985) 65.

${ }^{4}$ Eric J. Hobsbawm, Nationen und Nationalismus. Mythos und Realität seit 1780 (Frankfurt am Main 1991) 112-19 und 133-42, der aber auch die Grenzen des Sprachnationalismus aufzeigt.

${ }^{5}$ Viele anthropologische und soziologischen Untersuchungen über Ethnizität, die an heutigen Ethnien angestellt werden (etwa den vielen Minderheiten in den USA), gehen daher von einer prinzipiellen Unterscheidung von Nation (als Gemeinschaft der Staatsbürger) und Ethnie (als, zumindest subjektiv empfundene, Abstammungsgemeinschaft) aus; siehe etwa Ethnicity - Theory and Experience, ed. Nathan Glazer/Daniel P. Moynihan (Cambridge, Mass./London, 1975); Thomas H. Eriksen, Ethnicity and Nationalism: Anthropological Perspectives (London 1993); Ethnic Identity. Creation, Conflict and Accommodation, ed. Lola Romanucci-Ross/ George A. de Vos (Walnut Creek/London 1995); Ethnicity, ed. John Hutchinson/Anthony D. Smith (Oxford/ New York 1996). Das vernachlässigt jedoch das hohe ethnische Potential der Nationsbildung, siehe etwa Anthony D. Smith, The Ethnic Origins of Nations (London 1986).
} 
zungen, das Verhältnis zwischen Sprache und ethnischer Identität über lange Dauer hinweg zu verfolgen. In der Epoche zwischen etwa 400 und 1000 werden in Europa sowohl eine Vielzahl neuer Völker und Staaten als auch zahlreiche neue Sprachen greifbar. Dieses ,Laboratorium beschleunigter ethnischer Prozesse war Gegenstand des Wittgenstein-Preis-Projektes „Ethnische Identitäten im frühmittelalterlichen Europa“, in dessen Zusammenhang auch der vorliegende Band entstanden ist. ${ }^{6}$

Ethnische Prozesse finden ebenso wie Sprachwandel natürlich ständig statt; das führt zur Herausdifferenzierung von ethnischen Gruppen und von neuen Dialekten und Sprachen. ${ }^{7}$ Doch ist die historische Beschreibung dieser Prozesse problematisch. Was macht eine ethnische Gruppe zu einem eigenen Volk, und wodurch wird ein Dialekt innerhalb eines Sprachkontinuums zu einer distinkten Sprache? Wann wird etwa aus dem Lateinischen das Altfranzösische, oder: ist das Schwyzerdütsch bereits eine eigene Sprache? Die Entwicklung von Völkern und Sprachen ist ja nie abgeschlossen, ihre Genese ist daher Definitionsfrage. Dieses methodische Problem stellt sich sowohl bei der ,Ethnogenese wie bei der ,Glottogenese', allerdings in etwas unterschiedlicher Form; es wird verschärft dadurch, dass die beiden Prozesse in der modernen Forschung lange Zeit zu leicht in eins gesetzt wurde und daher unreflektiert von einem auf das andere geschlossen wurde. Für die Herausbildung von Sprachen lassen sich (falls ihre Formen einigermaßen gut bekannt sind) vielerlei linguistische Kriterien finden. Das ist bei Völkern wesentlich schwieriger; objektive Kriterien können Anhaltspunkte liefern, erlauben aber nicht immer eine Aussage über das jeweilige Zugehörigkeitsbewusstsein, gerade in Zeiten beschleunigten Wandels. Weil aber die Selbstzuordnung und die Fremdwahrnehmung für die Wirksamkeit ethnischer Identitäten eine wesentliche Rolle spielen, hinterlassen sie in den Quellen ihre Spuren. ${ }^{8}$ Gemeinsamkeiten und Unterschiede der Sprache werden bei der alltäglichen Verständigung sicherlich wahrgenommen; aber es ist durchaus möglich, damit pragmatisch umzugehen, ohne daraus ein stabiles Gruppenbewusstsein abzuleiten. In den schriftlichen Quellen findet das dann nicht unbedingt einen Niederschlag. Sprachliches Distanzbewusstsein kann zudem schon an kleinen Unterschieden ansetzen, die weit unterhalb der von der Philologie angenommenen Sprachgrenzen liegen.

Die Rede von der Sprache und den Völkern hat in den meisten Texten des europäischen Mittelalters eine andere Bedeutung. Dabei geht es um ihre Zeichenhaftigkeit, um ihre symbolische Bedeutung in der Ordnung der Welt. Zwei übermächtige Deutungssysteme prägten den mittelalterlichen Diskurs. Erstens repräsentierten im klassischen Weltbild Latein und Griechisch als kulturelle Codes die Überlegenheit der mediterranen Zivilisation über die Barbaren, was mit dem Vorrang des politisch verfassten Populus (bzw. der Polis) über barbarische Gentes bzw. Ethnē einherging. Rhetorik und Grammatik regulierten den Gebrauch der Sprachen und sorgten für ein hohes Maß an Reflexion über Struktur, Gebrauch und gesellschaftliche Bedeutung der Sprache. Wenn es also in unseren lateinischen Quellen des Frühmittelalters um Sprache geht, ist ein wenn auch unterschiedliches Maß an Reflexionsfähigkeit vorauszusetzen. Der zweite Diskurs ist der jüdisch-christliche, der in der Bibel ein umfangreiches Repertoire an Deutungsmöglichkeiten aller Gemeinschaftsformen zur Verfügung hatte. Hier ging es vor allem um die Rolle der Völker, vom auserwählten Volk Israel über die feindlichen Gentes zu den Völkern des Neuen Testamentes, deren Bekehrung eine heilsgeschichtliche Funktion hatte. Babel und Pfingsten boten ebenso ambivalente wie symbolisch aufgeladene Schlüssel zur Bedeutung der Sprachvielfalt. ${ }^{9}$ Die Äußerungen christlicher Autoren über Sprachen und Völker geben auch deshalb nicht unbedingt unverstellte Wahrnehmungen wieder.

\footnotetext{
${ }^{6}$ Die Verleihung des Wittgenstein-Preises durch den FWF im Jahr 2004 erlaubte die Durchführung eines Großprojektes, das 2005-10 am Institut für Mittelalterforschung der Österreichischen Akademie der Wissenschaften sowie an der Universität Wien angesiedelt war. Die Fertigstellung dieses Bandes stand bereits im Zusammenhang des durch den 2010 verliehenen ERC Advanced Grant ermöglichten Projektes „Social Cohesion, Identity and Religion in Europe, 400-1200“.

${ }^{7}$ Vgl. Entstehung von Sprachen und Völkern, ed. Per Sture Ureland (Linguistische Arbeiten 162, Tübingen 1985).

${ }^{8}$ Walter Pohl, Introduction. Strategies of identification: a methodological profile, in: Strategies of Identification. Ethnicity and Religion in Early Medieval Europe, ed. Walter Pohl/Gerda Heydemann (Turnhout, im Druck).

9 Arno Borst, Der Turmbau von Babel. Geschichte der Meinungen über Ursprung und Vielfalt der Sprachen und Völker, 1-5 (Stuttgart 1957-1965, München $\left.{ }^{2} 1995\right)$; Zwischen Babel und Pfingsten - Sprachdifferenzen und Gesprächsverständigung in der Vormoderne (8.-16. Jh.) / Entre Babel et Pentecôte. Différences linguistiques et communication orale avant la modernité (VIIIe - XVIe siècle), ed. Peter von Moos (Gesellschaft und individuelle Kommunikation in der Vormoderne 1, Wien/Berlin 2008).
} 


\section{FORSCHUNGSANSÄTZE UND METHODISCHE PROBLEME}

Über die Rolle der Sprache im Frühmittelalter wird selbstverständlich intensiv und auch interdisziplinär geforscht. Das vor kurzem nach 40 Jahren abgeschlossene Reallexikon für Germanische Altertumskunde bietet einen breiten Überblick über den Forschungsstand in interdisziplinärer Zusammenschau. ${ }^{10}$ Das Großprojekt ,Nomen et gens' untersucht seit langem die Identitätswirksamkeit von Personennamen; es ist zu hoffen, dass in absehbarer Zeit abschließende Befunde publiziert werden. ${ }^{11}$ Wolfgang Haubrichs hat in vorbildlicher Weise dazu beigetragen, aus germanistischer Sicht die verstreuten Befunde über die Rolle der Sprache in den nachrömischen Regna in zahlreichen Einzelstudien zusammenzutragen und zu überprüfen. ${ }^{12}$ Anknüpfend an derartige Untersuchungen kann die kaum behandelte Frage von Sprache und Identität im Frühmittelalter grundsätzlicher gestellt werden. ${ }^{13}$ Wie wirkten sich sprachliche Differenzen und Gemeinsamkeiten auf ethnische Identitäten im Frühmittelalter aus? Während in der frühgeschichtlichen Archäologie derzeit recht kontroversiell die ethnische Interpretation von Bodenfunden diskutiert wird, ${ }^{14}$ ist eine Methodendiskussion über die ethnische Interpretation frühmittelalterlicher philologischer Befunde bisher weitgehend ausgeblieben. ${ }^{15}$ Der vorliegende Band enthält dazu grundlegende Überlegungen, wobei durchaus verschiedene Akzente gesetzt werden. Vielleicht lassen sich auf diese Weise die polemischen Töne der archäologischen Debatten vermeiden.

Dass eine solche Diskussion nötig ist, soll hier am Beispiel zweier Arbeiten führender Historiker anderer Epochen gezeigt werden, die zu ganz unterschiedlichen Schlussfolgerungen kommen. Robert Bartlett hat in sein eindrucksvolles Buch „The Making of Europe, 950-1350“ ein Kapitel über ,Language“ als Aspekt der „Race Relations on the Frontiers of Latin Europe“ aufgenommen, das mit der apodiktischen Feststellung beginnt: „Language had a particularly important role in defining nationality“. ${ }^{16}$ Worauf stützt sich diese Beobachtung? Bartletts Belege dazu sind spätmittelalterlich und kommen so gut wie ausschließlich aus zwei geographischen Bereichen, nämlich aus Böhmen und von den Britischen Inseln. Beides sind aber sehr spezielle Fälle. Die Chroniken des Dalimil und Peters von Zittau aus der ersten Hälfte des 14. Jahrhunderts zum Beispiel, die er heranzieht, sind unter sehr spezifischen Umständen entstanden. Ihre Interpretation in der Literatur beruht immer noch

${ }^{10}$ RGA, 2. Aufl. 1-35 (Berlin/New York 1968-2008).

${ }^{11}$ Siehe den Beitrag von Dieter Geuenich in diesem Band sowie Horst Ebling/Jörg Jarnut/Gerd Kampers, Nomen et gens: Untersuchungen zu den Führungsschichten des Franken-, Langobarden-, und Westgotenreiches im sechsten Jahrhundert, in: Francia 8 (1980) 687-745; Nomen et gens. Zur historischen Aussagekraft frühmittelalterlicher Personennamen, ed. Dieter Geuenich/Wolfgang Haubrichs/Jörg Jarnut (RGA, Erg. Bd. 16, Berlin/New York 1997); Person und Name. Methodische Probleme bei der Erstellung eines Personennamenbuchs des Frühmittelalters, ed. Dieter Geuenich/Wolfgang Haubrichs/Jörg Jarnut (RGA, Erg. Bd. 32, Berlin/New York 2002).

12 Siehe den Beitrag von Wolfgang Haubrichs in diesem Band, sowie u.a. Wolfgang Haubrichs, Sprachliche Differenzen und Kongruenzen zwischen Sachsen und Franken innerhalb der ,Westgermania', in: Studien zur Sachsenforschung 12 (1999) 123-142; ders., Identität und Name, Akkulturationsvorgänge in Namen und die Traditionsgesellschaften des frühen Mittelalters, in: Die Suche nach den Ursprüngen. Von der Bedeutung des frühen Mittelalters, ed. Walter Pohl (Forschungen zur Geschichte des Mittelalters 8, Wien 2004) 85-105; ders., Amalgamierung und Identität - Langobardische Personennamen in Mythos und Herrschaft, in: Die Langobarden - Herrschaft und Identität, ed. Walter Pohl/Peter Erhart (Wien 2005) 67-99; ders., Baiern, Romanen und andere. Sprachen, Namen, Gruppen südlich der Donau und in den östlichen Alpen während des frühen Mittelalters, in: Zeitschrift für bayerische Landesgeschichte 69 (2006) 395-465.

13 Allgemein siehe Joanna Thornborrow, Language and identity, in: Language, Society and Power. An Introduction, ed. Linda Thomas/Ishtla Singh/Shân Wareing (London 2004) 158-172; Sprachidentität - Identität durch Sprache, ed. Nina Janich/Christiane Thim-Mabrey (Tübingen 2003); für weitere Literatur siehe den Beitrag von Wolfgang Haubrichs in diesem Band.

${ }^{14}$ Siehe dazu Archaeology of Identity - Archäologie der Identität, ed. Walter Pohl/Mathias Mehofer (Forschungen zur Geschichte des Mittelalters 17, Wien 2010).

${ }^{15}$ Siehe jedoch Haubrichs, Baiern, Romanen und andere; sowie Language of Religion - Language of the People. Medieval Judaism, Christianity and Islam, ed. Ernst Bremer/Jörg Jarnut/Michael Richter/David Wasserstein (MittelalterStudien 11, München 2006); Hans-Werner Goetz, Gentes et linguae. Völker und Sprachen im Ostfränkisch-deutschen Reich in der Wahrnehmung der Zeitgenossen, in: Theodisca. Beiträge zur althochdeutschen und altniederdeutschen Sprache und Literatur in der Kultur des frühen Mittelalters, ed. Wolfgang Haubrichs/Ernst Hellgardt/Reiner Hildebrandt/Stephan Müller/Klaus Ridder (Berlin/New York 2000) 290-312.

${ }^{16}$ Robert Bartlett, The Making of Europe. Conquest, Colonization and Cultural Change 950-1350 (Princeton 1993) 198204, hier 198. 
auf der nationalistischen Geschichtsschreibung, die darin Zeugnisse eines sogenannten ,Proto-Nationalismus ${ }^{6}$ sehen wollte; eine neue Kontextualisierung steht aus. ${ }^{17}$ Als die Przemysliden ausstarben und die Dynastie der Luxemburger an die Macht kam, lieferten die beiden (sehr unterschiedlich angelegten) Werke die intellektuellen Ressourcen eines auch sprachlich fundierten Selbstbewusstseins der (durchaus zweisprachigen) böhmischen Eliten und gaben Ratschläge für das neue Regime. In England wiederum, wo durch die lange französisch-englische Zweisprachigkeit das Bewusstsein für die kulturelle und politische Bedeutung sprachlicher Hegemonie geschärft war, ging die Expansion nach Wales, Schottland und Irland einher mit bewusster Anglisierungspolitik; so konnten englische Gesandte an der Kurie 1381 über irische Konflikte sagen, dass aus der Unterschiedlichkeit der Sprachen Kriege und diverse Konflikte entstanden seien. ${ }^{18}$

Bartlett verallgemeinert diese Bespiele zugespitzten politischen Gebrauchs von sprachlichem Distanzbewusstsein für das ganze Mittelalter. Gerade im Fall der Erforschung ethnischer und nationaler Identitäten sollten wir methodisch aber nicht so vorgehen, denn die Identitätswirksamkeit und politische Bedeutung ethnischer und sprachlicher Abgrenzungen war in unterschiedlichen Kontexten eben sehr verschieden. Die Geschichtsforschung kann und muss hier zu (im Sinn der Wissenschaftstheorie) wesentlich informationsgesättigteren Ergebnissen kommen als die soziologische Typologie und Modellbildung. Wir müssen daher zunächst danach trachten, die recht unterschiedliche Rolle der Sprache bei Identitätsbildung und Abgrenzung gegen andere möglichst spezifisch nach Raum und Zeit zu ordnen. So tut das auch Peter Burke in seiner Studie ,Sprache und Identität in Italien in der frühen Neuzeit", wo er den wachsenden Gebrauch überregionaler Varianten des Italienischen gegenüber dem regionalen Dialekt bei den Oberschichten skizziert; doch dabei konkurrenzierten sich lange die eklektische Hofsprache ,cortegiano' und das Toskanische, im 18. Jahrhundert auch das Französische. ${ }^{19}$ Erst gegen Ende des 18. Jahrhunderts findet sich ein deutlicher Bezug zwischen italienischem Zugehörigkeitsbewusstsein und der „lingua nazionale“. Zur selben Zeit stilisierte Johann Gottfried Herder in seiner Abhandlung „Über den Ursprung der Sprache“ (1772) die Sprache als nationales Identitätsmerkmal par excellence - mit nachhaltiger Wirkung auf die weitere Forschungsgeschichte. ${ }^{20}$

Die Philologie des 19. Jahrhunderts, etwa die Germanistik der Brüder Grimm, projizierte die Identifikation von Sprache und Nation in die Vergangenheit ebenso wie in die Zukunft. Die nationalen Literaturgeschichten sind erst heute dabei, sich von diesen linearen Geschichtskonstruktionen freizumachen. Der vorliegende Band bietet dafür eine Reihe von Beispielen, von denen hier nur einige genannt seien. Wie Uta Goerlitz zeigt, wurde etwa die frühmittelhochdeutsche Kaiserchronik aus der Zeit um 1150 lange als Dokument eines erwachenden deutschen Nationalgefühls interpretiert. Doch schreibt die Chronik den verschiedenen Völkern des Reiches eine ganz unterschiedliche Herkunft zu: Die Bayern kamen aus Armenien, die Franken aus Troja, die Sachsen stammten von den Heeren Alexanders des Großen ab, und die Schwaben hießen nach dem berge Swêro. ${ }^{21}$ Auch die Beiträge von Michel Banniard und Roger Wright gehen bei der Deutung des Übergangs vom Spätlatein zu den romanischen Sprachen neue Wege. Banniard ersetzt die gängige Vorstellung, das Altfranzösische habe sich im Gegensatz zur lateinischen Schriftsprache aus einem gesprochenen ,Vulgär-Latein ent-

${ }^{17}$ Geplant sind diese Untersuchungen im ERC-Projekt von Pavlína Rychterová, ,The Origins of the Vernacular Mode', der ich auch diese Hinweise verdanke. Immer noch die beste Studie ist František Šmahel, The Idea of the Nation in Hussite Bohemia. An Analytical Study of the Ideological and Political Aspects of the National Question in Hussite Bohemia from the End of the $14^{\text {th }}$ to the Eighties of the $15^{\text {th }}$ Century, in: Historica 16 (1969) 143-247; 17 (1969) 93-197; zweite, überarbeitete Auflage: ders., Idea národa v husitských Čechách (Praha 1999). Doch geht auch Šmahel von einem eher statischen Nationsbegriff aus.

${ }^{18}$ Edouard Perroy, L’Angleterre et le Grande Schisme d'Occident (Paris 1933) 394f.; Bartlett, The Making of Europe 198. Grundlegend zur Entwicklung des Verhältnisses von Englisch und Französisch im Spätmittelalter: Ardis Butterfield, The Familiar Enemy. Chaucer, Language and Nation in the Hundred Years War (Oxford 2009).

19 Peter Burke, Sprache und Identität im Italien der frühen Neuzeit, in ders., Reden und Schweigen. Zur Geschichte sprachlicher Identität (Berlin 1994) 7-30; siehe auch ders., Wörter machen Leute. Gesellschaft und Sprachen im Europa der frühen Neuzeit (Berlin 2006).

${ }^{20}$ Leerssen, National Thought 97-101.

${ }^{21}$ Die Kaiserchronik eines Regensburger Geistlichen (ed. Edward Schröder, MGH Deutsche Chroniken 1, 1, Hannover 1892) v. 271-454. Siehe auch Uta Goerlitz, Literarische Konstruktion (vor-)nationaler Identität seit dem Annolied. Analysen und Interpretationen zur deutschen Literatur des Mittelalters (11.-16. Jahrhundert) (Quellen und Forschungen zur Literatur- und Kulturgeschichte 45, Berlin/New York 2007). 
wickelt, durch ein komplexeres Modell verschiedener Sprachniveaus, die sich im geschriebenen Latein der Karolingerzeit niederschlagen; vieles, was die Philologen bislang einfach als ,verderbtes ${ }^{6}$ Latein betrachtet haben, wird damit als Zeugnis einer lebendigen Sprachentwicklung lesbar. ${ }^{22}$ Ähnliches lässt sich auf der Iberischen Halbinsel verfolgen, wo in der Westgotenzeit trotz ethnischer Vielfalt rasch relative spätlateinische linguistische Einheit erreicht wurde. ${ }^{23}$ Peter Štih diskutiert die philologischen Versuche, schon für das Mittelalter eine alpenslawische oder slowenische Sondersprache im Ostalpenraum nachzuweisen; doch lässt sich zeigen, dass im Mittelalter selbst bei wachsendem Distanzbewusstsein unter den sich herausbildenden slawischen Einzelvölkern, etwa zwischen Böhmen und Polen, sprachliche Unterschiede erst relativ spät identitätsrelevant wurden. ${ }^{24}$ Dieter Geuenich zeigt am Beispiel des Alemannischen, wie eine heutige regionale Identität aus der Rückprojektion dialektaler Besonderheit auf ein frühmittelalterliches Volk eine Stütze finden kann. Nur wo die so lange selbstverständliche Gleichsetzung von Sprache und ethnischer Identität aufgegeben wird, das wird an diesen Beispielen deutlich, lässt sich ein differenziertes Bild vom jeweils spezifischen Zusammenhang zwischen Sprachentwicklung und frühmittelalterlicher Identitätsbildung gewinnen.

\section{PERSPEKTIVEN DER ERFORSCHUNG FRÜHMITTELALTERLICHER IDENTITÄTEN}

Ist, wonach wir suchen, noch dasselbe wie vor einigen Jahrzehnten? Oder verlangt der rasche Wandel des begrifflichen Rahmens nach neuer Verständigung über Weg und Ziel interdisziplinären Forschens über Großgruppen und Zugehörigkeiten im Frühmittelalter? Noch nach 1945 suchte man nach Völkern und ihren Kulturen; seit Wenskus' und Wolframs bahnbrechenden Werken nach Stammesbildungen und Ethnogenesen sowie ihren kulturellen Spuren; ${ }^{25}$ seit den neunziger Jahren zunehmend allgemeiner nach ethnischen (und anderen) Identitäten und ihrer kulturellen Konstruktion. Der Begriff Identität wird recht unterschiedlich gebraucht und ist deshalb auch zuweilen als zu schwammig verworfen worden. ${ }^{26}$ Sicherlich, wer sich unter ,ethnischer Identität' nichts anderes vorstellt als man vor 60 Jahren unter der ,Zugehörigkeit zu einem Volk' verstand, der braucht den Begriff gar nicht erst zu verwenden. Ethnische Identität ist das Resultat nachhaltiger Identifikationen, und zwar auf drei verschiedenen Ebenen: das Bekenntnis von Einzelnen oder kleinen Gruppen zu einer bestimmten ethnischen Formation; die Selbstvergewisserung und Selbstdarstellung dieses ethnischen Verbandes; und die Wahrnehmung und Kategorisierung durch Außenstehende. ${ }^{27}$ Die Stabilität ethnischer Gruppen ist umso größer, je weitgehender diese drei Ebenen längerfristig zur Deckung kommen. Doch gelingt das nie völlig, sodass das Gleichgewicht zwischen Selbst- und Fremdzuordnung immer dynamisch bleibt, eine Dynamik, die sich unter bestimmten Bedingungen rasch verstärken kann.

Aus meiner Sicht bietet der Identitätsbegriff folgende methodische Vorteile:

1. Er erlaubt es, sowohl die persönliche als auch die soziale Identität in den Blick zu nehmen, also Zugehörigkeitsbewusstsein und Bezugsgruppe, und erfasst damit eine Schnittstelle zwischen Individuum und Gesellschaft. Dieses Spannungsverhältnis ist auch für den Bezug zwischen Sprache und Identität bedeutungsvoll; zum Beispiel sind Personennamen ein wesentliches Element sozialer Verortung, und doch leisten sie Zuordnung wie Abgrenzung in recht unterschiedlichen Ausprägungen.

${ }^{22}$ Siehe auch Michel Banniard, Viva voce. Communication écrite et communication orale du IVe au IXe siècle en Occident Latin (Paris 1992).

${ }^{23}$ Roger Wright, Early Ibero-Romance (Newark 1995); ders., A Sociophilological Study of Late Latin (Turnhout 2003).

${ }^{24}$ František Graus, Die Nationenbildung der Westslawen im Mittelalter (Nationes 3, Sigmaringen 1980). In Böhmen und Polen begann dieser Prozess im 14. Jahrhundert, für die slowenische Sprache war die Verschriftung in der Reformationszeit prägend.

${ }^{25}$ Reinhard Wenskus, Stammesbildung und Verfassung. Das Werden der frühmittelalterlichen gentes (Köln 1961/Wien ${ }^{2} 1977$ ); Herwig Wolfram, Die Goten. Von den Anfängen bis zur Mitte des sechsten Jahrhunderts. Entwurf einer historischen Ethnographie (München ${ }^{5} 2010$ ); vgl. Walter Pohl, Tradition, Ethnogenese und literarische Gestaltung: eine Zwischenbilanz, in: Ethnogenese und Überlieferung. Angewandte Methoden der Frühmittelalterforschung, ed. Karl Brunner/ Brigitte Merta (Veröffentlichungen des Instituts für Österreichische Geschichtsforschung 31, Wien 1994) 9-26.

${ }^{26}$ Z.B. Lutz Niethammer, Kollektive Identität. Heimliche Quellen einer unheimlichen Konjunktur (Reinbek 2000); Rogers Brubaker/Frederick Cooper, Beyond ,identity‘, in: Theory and Society 29 (2000) 1-47.

${ }^{27}$ Dazu und zum Folgenden: Walter Pohl, Aux origines d'une Europe ethnique: Identités en transformation entre antiquité et moyen âge, in: Annales: Histoire, Sciences sociales 60/1 (2005) 183-208; ders., Strategies of identification. 
2. Er hebt die ethnische nicht prinzipiell ab von anderen Formen der Identität, zum Beispiel Geschlecht, Alter, Familie, Religion, sozialem Status etc. Damit wirft er zwar die Frage auf, was denn nun eigentlich spezifisch für ethnische Identität sei - wie verhält sie sich etwa zu nationalen, lokalen, kulturellen, politischen Identitäten? Doch ermöglicht er zugleich den Vergleich und die Untersuchung von Interferenzen.

3. Der dritte terminologische Vorteil hat sich paradoxerweise in Spannung zur eigentlichen Begriffsbedeutung durchgesetzt: Identität beschreibt (so der inzwischen weitgehende Konsens in den Kultur- und Sozialwissenschaften) eben nicht das Identisch-sein, sondern den nie abgeschlossenen Prozess der Identitätsbildung, bei dem Individuum wie soziale Gruppen unterschiedliche Identitäten in Balance halten müssen. Unter ,Identität' verstehen wir also einen offenen Prozess der Identitätsbildung und der Identifikationen; oder, um es eleganter mit Zygmunt Baumann zu sagen: „Identity, though ostensibly a noun, behaves like a verb, albeit a strange one to be sure: it appears only in the future tense.“"28

4. Identität kann als Ergebnis von Kommunikation verstanden werden. Dadurch werden vergangene ,Texte der Identität ${ }^{\star}$ als Spuren von Verhandlungen über Identität interpretierbar, nicht bloß als Widerspiegelungen von unabhängig davon existierenden Gemeinschaften. Auch wenn solche Kommunikation oft symbolischen Charakter hat, bedarf sie zumeist der Sprachform, wobei Ethnonymen eine Schlüsselrolle in der Kommunikation über spezifische ethnische Identifikationen zukommt.

5. Der Identitätsbegriff beinhaltet neben Selbst- und Fremdbezeichnung auch Wunschbilder, Entwürfe und Vorstellungen. ${ }^{29}$ Diese verweisen nicht bloß auf Phantasiewelten, sondern sind ein wesentlicher Bereich gesellschaftlicher Diskurse, die erwartungsprägend und handlungsleitend sein können, durchaus im Sinn der von Hans-Werner Goetz propagierten Vorstellungsgeschichte. ${ }^{30}$ In diesem Zusammenhang könnte man ,Ethnizität' (als Einteilungsprinzip der sozialen Welt auf der diskursiven Ebene) unterscheiden von ,ethnischer Identität‘ (als jeweiliges Ergebnis der Kommunikation über ethnische Identifikationen).

6. Identität ist daher zugleich mehr und weniger als die Zugehörigkeit zu einer konkreten sozialen Gruppe, deren Gestalt in Identitätsbildungsprozessen ja auch immer wieder auf dem Spiel steht. ${ }^{31}$ Persönliche Identität balanciert fast immer mehrere Referenzgruppen, wobei der Bezug unterschiedlich intensiv sein kann. Das muss, wenn wir von frühmittelalterlichen Völkern sprechen, mitgedacht werden: Wenn in den Quellen von ,den Franken' die Rede ist, so können damit einmal ein paar Hundert, ein andermal einige Tausend, dann wieder Hunderttausende gemeint sein. ${ }^{32}$ Das Gewicht (, salience in der anthropologische Literatur) dieser Identifikationen kann sehr unterschiedlich sein. ${ }^{33}$

${ }^{28}$ Zygmunt Baumann, From pilgrim to tourist - or a short history of identity, in: Questions of Cultural Identity, ed. Stuart Hall/Paul du Gay (London/Thousand Oaks 1996) 18-36, hier 19.

${ }^{29}$ Siehe den Beitrag von Herwig Wolfram in diesem Band.

${ }^{30}$ Hans-Werner Goetz, Vorstellungsgeschichte. Gesammelte Schriften zu Wahrnehmungen, Deutungen und Vorstellungen im Mittelalter, ed. Anna Aurast/Simon Elling/Bele Freudenberg/Anja Lutz/Steffen Patzold (Bochum 2007). Siehe auch den Beitrag von Hans-Werner Goetz in diesem Band.

${ }^{31}$ Identität ist daher nicht einfach als Gruppenzugehörigkeit zu beschreiben. Eine fundamentale Kritik an der Verdinglichung der sozialen Gruppe in den Sozialwissenschaften findet sich bei Rogers Brubaker, Ethnicity without Groups (Cambridge-Massachusetts/London 2004); Rogers Brubaker, Ethnicity without groups, in: Facing Ethnic Conflicts Towards a New Realism, ed. Andreas Wimmer/Richard Goldstone/Donald Horowitz/Ulrike Joras/Conrad Schetter (Lanham-Maryland 2004) 34-42. Ähnliche, aber abgewogenere Überlegungen bei Richard Jenkins, Rethinking Ethnicity. Arguments and Explorations (Los Angeles etc. ${ }^{2} 2008$ ).

32 Ian N. Wood, Defining the Franks: Frankish origins in early medieval historiography, in: Concepts of National Identity in the Middle Ages, ed. Simon Forde/Lesley Johnson/Alan V. Murray (Leeds Texts and Monographs. New series 14, Leeds 1995) 47-57; Walter Pohl, Zur Bedeutung ethnischer Unterscheidungen in der frühen Karolingerzeit, in: Studien zur Sachsenforschung 12, ed. Hans-Jürgen Hässler (Oldenbourg 1999) 193-208; Helmut Reimitz, Omnes Franci. Identifications and identities of the early medieval Franks, in: Franks, Northmen, and Slavs: Identities and State Formation in Early Medieval Europe, ed. Ildar H. Garipzanov/Patrick J. Geary/Przemysław Urbańczyk (Turnhout 2008) 51-70.

33 ,Salience': Stephen Worchel/Jonatan Iuzzini/Dawna Coutant/Manuela Ivaldi, A multidimensional model of identity: relating individual and group identities to intergroup behaviour, in: Social Identity Processes, ed. Dora Capozza/Rupert Brown (London/Thousand Oaks, 2000) 15-32, bes. 26-29; ,degrees': Eriksen, Ethnicity and Nationalism 41-44; Jan Ass- 
Wir sollten daher zunächst prinzipiell unterscheiden zwischen der Menge aller Sprecher der Sprache X, einer nach einem modernen Kriterium klassifizierten Bevölkerungsgruppe, und dem Volk X, das oft denselben Namen wie die Sprache trägt und eine zeitgenössisch wahrgenommene, aber kaum abgrenzbare Einheit bezeichnet. Doch öffnet der oben skizzierte Identitätsbegriff ein viel breiteres Untersuchungsfeld: Denn es gibt ja keine Identifikation ohne Kommunikation, und Sprache ist bei weitem das bedeutendste Medium, in dem Identitäten entworfen, behauptet, verhandelt oder beschrieben werden. Sie gibt den Kommunikationsrahmen vor, in dem Identitäten sich durchsetzen müssen.

Daraus ergibt sich für die Frage nach ,Sprache und Identität' zunächst, dass Sprache das wesentliche Medium der ,Verhandlungen“ über ethnische Identität ist. Kommunikation über die Zugehörigkeit zu sozialen Gruppen bedarf (auch) sprachlicher Codes, in denen die Existenz dieser Gruppe als möglich, wirklich und legitim dargestellt wird und sie von anderen ähnliche Gruppen abgegrenzt wird. Diese Codes sind vor allem zwischen den Mitgliedern einer ethnischen Gruppe von Bedeutung, bei denen sie das Zusammengehörigkeitsbewusstsein verstärken sollen. Doch ist die Sprache der symbolischen Identifikation nicht notwendiger Weise deckungsgleich mit der Alltagssprache. Die imperiale römische Identität wurde zum Beispiel, unabhängig von der Muttersprache dieser ,Römer', über das Lateinische und Griechische transportiert. Ähnlich war das mit der christlichen Identität, für die zusätzlich der symbolische Bezug auf das Hebräische bedeutsam war. In den frühmittelalterlichen Regna schafften die lateinische Staatssprache und das Latein der Kirche prestigeträchtige Medien der Identifikation, die keineswegs nur supra-gentile Zuordnungen ermöglichten. Im Gegenteil, die Besonderheit, ja Auserwähltheit der jeweiligen Gens ließ sich auch sehr gut im römischen wie im christlichen Diskurs formulieren. Das Alte Testament bot außerordentlich attraktive ethnische Identifikationsmuster, die sowohl zur Selbstüberhöhung als Gens als auch zur Abgrenzung von den anderen Gentes dienen konnten. ${ }^{34}$ Die Verhandlungen zwischen individuellen und kollektiven Selbstzuordnungen und Fremdwahrnehmungen bedurften also regelmäßig der Übersetzung, zumindest zwischen der diskursleitenden lateinischen Staats- und Kirchensprache und der lingua theodisca der einfachen Angehörigen vieler frühmittelalterlicher Völker. ${ }^{35}$ Die gotische Origo gentis aus Skandinavien ist bei Jordanes auf Latein überliefert; der Text könnte durchaus auf den römischen Senator im Ostgotenreich, Cassiodor, als ,Cultural broker ${ }^{6}$ zurückgehen. ${ }^{36}$ Auf gotisch dagegen ist Wulfilas Bibelübersetzung in der Prachthandschrift des Codex Argenteus erhalten. Beide Texte waren zentral für die gotische Identität im Ostgotenreich Theoderichs, und beide waren das erst durch eine Übersetzung geworden. Für die Identitätswirksamkeit der Sprache konnte das nicht ohne Folgen bleiben.

\section{FRÜHMITTELALTERLICHE VORSTELLUNGEN VON DER BEDEUTUNG VON SPRACHEN UND VÖLKERN}

Antike und mittelalterliche Gelehrte nennen, ähnlich wie viele moderne Autoren, habitus, arma, vestes und fast immer auch lingua, manchmal auch lex als Kriterien ethnischer Zugehörigkeit. Doch sind das abstrakte Vorstellungen, die zwar bis zu einem gewissen Grad evident gemacht werden können, aber eine tatsächliche Abgrenzung von Großgruppen nicht leisten können. Das hat ja schon Reinhard Wenskus gezeigt, und es ergibt sich auch aus dem archäologischen Befund bei Tracht und Bewaff-

mann, Das kulturelle Gedächtnis: Schrift, Erneuerung und politische Identität in frühen Hochkulturen (München 1992) 144-160, unterscheidet zwischen einer ,Grundstufe‘ und einer ,Steigerungsstufe‘ der ethnischen Identität.

${ }^{34}$ Walter Pohl, Disputed Identification: The Jews and the Use of Biblical Models in the Barbarian Kingdoms, in: Barbarians and Jews - Jews and Judaism in the Early Medieval West, ed. Yitzhak Hen (im Druck).

${ }^{35}$ Zur Problematik der Übersetzung im Mittelalter siehe z.B. The Medieval Translator. The Theory and Practice of Translation in the Middle Ages, Roger Ellis (Cambridge 1989); Rita Copeland, Rhetoric, Hermeneutics and Translation in the Middle Ages. Academic Traditions and Vernacular Texts (Cambridge 2001); Translation Theory and Practice in the Middle Ages, ed. Jeanette Beer (Kalamazoo 1997); Translatio or the Transmission of Culture in the Middle Ages and the Renaissance. Modes and Messages, ed. Laura Hollengreen (Turnhout 2008).

36 Iordanes, Getica 25-28 (ed. Theodor Mommsen, MGH AA 5, 1, Berlin 1882) 60f.; siehe dazu Herwig Wolfram, Origo gentis (Goten), in: RGA, 2. Aufl. 22 (2003) 178-83, und ausführlicher ders., Gotische Studien. Volk und Herrschaft im frühen Mittelalter (München 2005) 83. Zu den ,Cultural brokers': Helmut Reimitz, Cultural brokers of a common past: History, identity and ethnicity in Gregory of Tours and the chronicles of Fredegar, in: Strategies of Identification. Ethnicity and Religion in Early Medieval Europe, ed. Walter Pohl/Gerda Heydemann (Turnhout, im Druck). 
nung. ${ }^{37}$ Die Schriftquellen des Frühmittelalters, zumindest vor der Karolingerzeit, bieten ebenfalls nur wenig Anhaltspunkte dazu, dass objektiven Kriterien wirklich zur Bestimmung ethnischer Zugehörigkeit benützt wurden. ${ }^{38}$ Bei der Sprache hängt das natürlich auch zusammen mit den in den Sprachwissenschaften wohlbekannten Problemen der Spracheinheit. Welche der verschiedenen Sprachschichten konnten in einer Zeit der Diglossie, der dialektalen Verschiedenheiten, der Mehrsprachigkeit und Auflösung territorialer Kontinua überhaupt identitätswirksam werden? Hier ist die Geschichtsforschung auf das Urteil der Philologien angewiesen. Viele Beispiele für sprachliches Distanzbewusstsein (wie sie etwa im Beitrag von Wolfgang Haubrichs zusammengestellt sind) beziehen sich auf relativ kleine Unterschiede im regionalen Gesichtskreis, wo die Spannung zwischen Vertrautheit und Fremdheit die Wahrnehmungen schärfte. Eine gänzlich fremde Sprache wiederum erleichterte zwar die eigene Abgrenzung, aber nicht notwendiger Weise die Unterscheidung verschiedener Fremdsprachen voneinander.

Welches Verständnis hatten Gebildete des Frühmittelalters vom Verhältnis von Sprache und Identität? Gerade lingua stellte für die christlichen Zeitgenossen eine Herausforderung dar. In der Bibel wird der Sprache große Bedeutung eingeräumt. In principium erat verbum, et verbum erat apud Deum, das ist zumindest ebenso deutlich auf Sprache bezogen wie logos in der griechischen Fassung. „The Christian God is modelled on language“, hat G. G. Harpham etwas zugespitzt geschrieben. ${ }^{39}$ Eine der nachhaltigsten Strafen des alttestamentarischen Gottes für die sündige Menschheit war demgemäß die babylonische Sprachverwirrung. Noch schärfer war allerdings eine andere Menschheitsstrafe, nämlich die Sintflut. Das Problem besteht nun darin, dass die Genealogie der Völker auf die eine Katastrophe zurückgeführt wird (nämlich aus den Nachkommen Noahs abgeleitet), die Entstehung der Sprachen aber aus der zweiten (dem Turmbau zu Babel). Arno Borst hat ja schon vor über einem halben Jahrhundert den unterschiedlichen Interpretationen dieser beiden Stellen ein dickes Buch gewidmet, seinen ,Turmbau von Babel ${ }^{6}{ }^{40}$ Allerdings hebt der Bibeltext den Widerspruch zum Teil wieder auf, indem Gott selbst von den Menschen beim Turmbau (Gen. 11, 6) sagt: „Seht nur, ein Volk sind sie und eine Sprache haben sie alle.“

Isidor von Sevilla, hier nicht wie sonst über weite Strecken gestützt auf Augustinus und Hieronymus, schließt folgerichtig: Ex linguis gentes, non ex gentibus linguae exortae sunt. ${ }^{41}$ Ethnische Abgrenzungen entstehen also als Folge beschränkter Verständigungsmöglichkeiten. Doch im Widerspruch dazu muss er, ähnlich wie Augustinus, auch feststellen: ${ }^{42}$ Initio autem quot gentes tot linguae fuerunt, deinde plures gentes quam linguae, quia ex una lingua multae sunt gentes exortae. In dieser Vorstellung haben die Sprachen also sozusagen eine genealogische, aber keine prinzipiell distinktive Funktion (da es ja viele gleichsprachige Gentes gibt). Gerade die „heiligen“ Sprachen Hebräisch, Griechisch und Latein stehen einer durchgehenden ethnischen Identifikation im Wege. Hebräisch war vor dem Turmbau die Sprache aller Menschen; das Griechische gliedert Isidor nach den klassischen Grammatikern in fünf Dialekte, partes, das Lateinische in vier Stadien der Sprachentwicklung. ${ }^{43}$ Beim Lateinischen musste Isidor auch besonders klar sein, dass viele der von ihm aufgezählten Gentes (etwa in Italien) dieselbe Sprache sprachen.

Im folgenden, langen Abschnitt über die einzelnen Völker kommt nur einmal die Sprache als Distinktionsmerkmal vor. Es geht dabei um die Germaniae gentes: Horum plurimae gentes, variae

\footnotetext{
37 Wenskus, Stammesbildung 93-102: „Die Ethnosbildung selbst geht gewöhnlich nicht von einem gemeinsamen Kulturbesitz aus“ (93).

38 Walter Pohl, Telling the difference - Signs of ethnic identity, in: Strategies of Distinction. The Construction of Ethnic Communities, 300-800, ed. Walter Pohl/Helmut Reimitz (The Transformation of the Roman World 2, Leiden/New York/Köln 1998) 17-69.

${ }^{39}$ Geoffrey G. Harpham, The Ascetic Imperative in Culture and Criticism (Chicago 1987) 17; zustimmend zitiert bei Averil Cameron, Christianity and the Rhetoric of Empire: The Development of Christian Discourse (Berkeley/Los Angeles/Oxford 1991) 6.

${ }^{40}$ Borst, Der Turmbau von Babel.

${ }^{41}$ Isidor, Etymologiae IX, 1, 14, ed. Lindsay. Ähnlich schon Claudius Marius Victor († 425/50) (Alethia III, $210 \mathrm{ff}$. [ed. Carl Schenkel, CSEL 16, Wien 1888]) 335-498, hier 414f.: gentem lingua facit. Siehe Borst, Turmbau 2, 414.

${ }^{42}$ Isidor, Etymologiae IX, 1, 1, ed. Lindsay.

${ }^{43}$ Isidor, Etymologiae IX, 1, 5-6, ed. Lindsay.
} 
armis, discolores habitu, linguis dissonae et origine vocabulorum incertae. ${ }^{44}$ Isidor exemplifiziert also gerade am Beispiel der Völker der Germania die Distinktion durch Sprache. Sicherlich, er bezieht sich damit auf längst vergangene Völker (er nennt Quaden, Brukterer, Chamaven und andere), und die Stelle kann nicht als Beleg für zeitgenössische Probleme der Verständlichkeit oder „,receptibilité“ dienen. Aber die Vorstellung einer, gemeingermanischen' Sprache war Isidor wie seinen Zeitgenossen fremd. Die Goten rechnete er ohnehin nicht zu den Germanen; sie gehören zum Steppenvölker-Komplex Skythen, Geten, Gog und Magog, aber er erwägt sogar ihre enge Verwandtschaft mit den Mauren, worauf der Völkername der Getuli hindeute. ${ }^{45}$ Das etymologische Prinzip, Verwandtschaft von Völkern aus Namensähnlichkeiten abzuleiten, durchkreuzt an dieser Stelle (wie auch sonst öfters) sowohl die biblische Völkergenealogie als auch Isidors eigene Prämisse, mehrere Völker seien jeweils aus einer Sprache hervorgegangen. Die Etymologie ist Isidors flexibelstes Instrument, um ethnische Identitäten zu erfassen. Die Namen werden wie schon bei Herodot von eponymen Königen und Heroen, Toponymen, körperlichen Besonderheiten, hervorstechenden Eigenschaften, Bräuchen oder von anderen Völkern abgeleitet. Etymologie ist Origo, wie schon der Titel sagt; in diesem Sinn ist die Gens aus der Sprache hervorgegangen wie die Welt aus dem Wort. Manche von Isidors Ableitungen mögen uns abwegig vorkommen; aber das ist ja bei der modernen Diskussion um die Etymologie des Bayernnamens auch nicht viel anders. ${ }^{46}$ Dahinter steht aber die historische Erfahrung von der Vielfalt der Formen und Entwicklungsgänge ethnischer Gruppen. Sprache, das war Isidor wohl bewusst, konnte dabei unterschiedliche Rollen spielen.

Dass Isidor das Wesentliche der Gentes von den Völkernamen her zu erfassen versucht, entspricht einer soziologischen Besonderheit ethnischer Identitäten. Unter den vielerlei Formen sozialer Identitäten sind nur wenige so stark an ein Nomen geknüpft und fügen sich dabei in eine flächendeckende Namenslandschaft ein ${ }^{47}$ (Dynastienamen etwa hatten lange nicht dieselbe Bedeutung). ${ }^{48}$ Zudem waren viele Völkernamen der Zeit sprechend, was Isidor allerdings nur ausnahmsweise (etwa bei den Langobarden) richtig erfasst. ${ }^{49}$ Der Name evoziert oft Geschichten (wie bei der Legende von der Namengebung der Langobarden ${ }^{50}$ ), oder er wird in abschätziger Weise interpretiert (etwa leiteten die Goten den Namen der Gepiden von gepanta, faul, $\mathrm{ab}^{51}$ ). Das Frühmittelalter suchte viel aus Namen herauszulesen. Wolfgang Haubrichs hat eindrucksvoll dargestellt, dass im Frühmittelalter sowohl die christliche als auch die germanische Personennamengebung starke und suggestive Bedeutungen bevorzugten, bis hin zum ,als König berühmten“ Cunincpert und zum „unerhörten“ bitheriophoren Wolfram, dem Wolfsraben. ${ }^{52}$ Andererseits ist der Zusammenhang von Personennamen und ethnischen Zugehörigkeiten dynamisch. Durch recht subtile Modulationen innerhalb der Namengebung einer Familie konnte Sprachwandel bei ethnischer Kontinuität elastisch aufgefangen werden, wie die Latinisierung germanischer Personennamen zeigt. Innerhalb derselben Familie und desselben Bestandes an Leitnamen konnte aber auch ethnischer und politischer Wandel ausgedrückt werden; ein Beispiel dafür sind die Übergänge zwischen angelsächsischen und skandinavischen Namenformen im Osten Englands im

\footnotetext{
${ }^{44}$ Isidor, Etymologiae IX, 2, 97, ed. Lindsay: ut Tolosates, Amsivari, Quadi, Tungri, Marcomanni, Bructeri, Camavi, Vangioni, Tubantes quorum inmanitas barbariae etiam in ipsis vocabulis horrorem quendam significat.

${ }^{45}$ Isidor, Etymologiae IX, 2, 118, ed. Lindsay.

${ }^{46}$ Doch steht dahinter wiederum die Vorstellung, jedes Volk zeichne sich eben durch seine propria collectio aus, wie Isidor bei der Definition des Begriffes gens hervorhebt.

${ }^{47}$ Siehe dazu die Beiträge von Jörg Jarnut, Dieter Geuenich und Corinna Bottglieri in diesem Band.

48 Vor 800 sind nur wenige zeitgenössische Dynastie- oder Sippennamen überliefert: Amaler und Balthen bei Jordanes, Merowinger einmal bei Fredegar und einmal im selben Zusammenhang im Liber Historiae Francorum (der Name ist uns so selbstverständlich geworden, dass wir uns gar nicht bewusst machen, wie dünn seine Überlieferung ist und dass er eben nicht zur historiographischen Erzählung benutzt wird), Agilolfinger (und die fünf bayerischen Genealogiae). Bei den Langobarden gibt die Königsliste des Edictus Rothari Dynastienamen bis hinauf zu Rothari selbst an, die Origo tut das nur für die Guginger. Alle diese Dynastien werden kaum je als Handlungsträger vorgestellt.

${ }^{49}$ Isidor, Etymologiae IX, 2, 95, ed. Lindsay.

${ }^{50}$ Walter Pohl, Origo gentis (Langobarden), in: RGA, 2. Aufl. 22 (2003) 183-88.

${ }^{51}$ Iordanes, Getica XVII 95, ed. Mommsen 82; Wolfram, Goten 37f. Siehe auch den Beitrag von Wolfgang Haubrichs in diesem Band.

${ }^{52}$ Haubrichs, Identität und Name, bes. 97.
} 
10. und 11. Jahrhundert. ${ }^{53}$ Namen konnten natürlich auch aus anderen Sprachen übernommen werden; vereinzelt ist ein Bewusstsein dafür bereits bei Zeitgenossen festzustellen, zum Beispiel bei Jordanes, der geradezu ein Namenskarussell zwischen den Völkern annimmt. ${ }^{54}$ Das Nomen und Gens-Projekt wird solche Zusammenhänge vielleicht klarer zeigen können. Explizit wird die Verbindung zwischen Personennamen und Volkszugehörigkeit in den Quellen der Zeit freilich kaum hergestellt.

Dass alle Regna vielsprachig waren, wird in den Quellen nur ausnahmesweise zum Thema. Spätlatein (oder frühromanisch), selbst in sich gegliedert, koexistierte mit germanischen Sprachen; andere kamen oft dazu. ${ }^{55}$ Im Reich König Oswalds, meint Beda, wurden die Sprachen der Briten, Pikten, Schotten und Angeln gesprochen. ${ }^{56}$ Als der Frankenkönig Guntram nach Orléans kam, so berichtet Gregor von Tours, wurde er von einer vielsprachigen Volksmenge empfangen: Et hinc lingua Syrorum, hinc Latinorum, hinc etiam ipsorum Iudaeorum in diversis laudibus variae concrepabat. ${ }^{57}$ Was zunächst positiv klingt, wird durch das wenig schöne Verb concrepare und gleich anschließend durch Guntrams Ausfälle gegen die Juden fragwürdig. Nicht die Rede ist übrigens von der Sprache der Franken. Die Vielsprachigkeit wurde offenbar ambivalent gesehen.

Das hat auch einen christlichen Hintergrund. Zwar hatte das Pfingstwunder die göttliche Strafe der Sprachenvielfalt zum Teil aufgehoben. Aber schon Paulus hat im Korintherbrief die Glossolalie in der Hierarchie der Charismata niedrig gereiht: „Wer in Zungen redet, erbaut sich selbst; wer aber prophetisch redet, baut die Gemeinde auf." ${ }^{658}$ Der Zungenrede fehlte das klar umrissene Wort; es sollte ja um das Verkünden gehen, nicht um bloßes Reden. Die Kirchenväter übernahmen diese eher ambivalente Haltung. Hieronymus erzählt in seiner Vita Hilarionis vom Fall eines Soldaten fränkischer Herkunft, dessen Besessenheit sich vor allem darin ausdrückte, dass er akzentfrei griechisch und syrisch sprechen konnte: Videres de ore barbaro, et qui francam tantum et latinam noverat, syra ad purum verbum resonare, ut non stridor, non aspiratio, non idioma aliquod palaestini deesset eloquii. ${ }^{59}$ Das war die Sprachkompetenz des Dämons, den der heilige Hilarion umgehend austrieb. In der präzisen Terminologie (stridor, aspiratio, idioma) demonstriert Hieronymus seine linguistische Bildung; er wusste gut über verschiedene Niveaus der Fremdsprachenkenntnis Beschied.

Wie ein Kommentar zum Korintherbrief wirkt es, was Gregor der Große in den Dialogi über eine Pestepidemie erzählt. Ein erkrankter Diener erfährt vor seinem Tod in einer Jenseitsvision, wer außer ihm aus dem Haushalt sterben muß. Damit man ihm glaubt, demonstriert er die auf seiner Himmelfahrt erworbene Beherrschung sämtlicher Sprachen, indem er mit seinem Herrn Griechisch und mit einem zufällig anwesenden bulgarischen Spatharius des Narses Bulgarisch spricht, ac si ex eadem fuisset gente generatus. Vor seinem Tod zerfleischt er sich noch die Arme mit den Zähnen (eigentlich ein Zeichen von Besessenheit); doch treffen alle seine Voraussagen ein. Gregors Dialogpartner wendet ein: Valde terribile est ut qui tale donum percipere meruit, tali etiam post hoc poena plecteretur. Doch Gregor meint nur: Timere magis quam discutere debemus. ${ }^{60}$ Das Sprachwunder hat nur dazu

${ }^{53}$ Das Beispiel verdanke ich Arnaud Lestremau, Paris (z.B. die Ketel-Sippe). Siehe auch John Insley, Pre-conquest personal names, in: RGA, 2. Aufl. 23 (2003) 367-96, bes. 381-90.

${ }_{54}$ Jordanes, Getica 9, ed. Mommsen 70: ne vero quis dicat hoc nomen a lingua Gothica omnino peregrinum esse, nemo qui nesciat animadvertat usu pleraque nomina gentes amplecti, ut Romani Macedonum, Greci Romanorum; Sarmatae Germanorum, Gothi plerumque mutuantur Hunnorum. Siehe auch den Beitrag von Hans-Werner Goetz in diesem Band.

${ }^{55}$ Siehe dazu die Beiträge von Wolfgang Haubrichs, Hans-Werner Goetz, Herwig Wolfram, Roger Wright und Michel Banniard in diesem Band.

${ }^{56}$ Beda, Historia ecclesiastica gentis Anglorum III, 6 (ed. Bertram Colgrave/Roger A.B. Mynors, Oxford Medieval Texts, Oxford 1969) 230; (übers. Günter Spitzbart, Texte zur Forschung 34, Darmstadt 1982) 223. Siehe den Beitrag von Anton Scharer in diesem Band.

${ }^{57}$ Gregor von Tours, Historiae VIII, 1 (ed. Bruno Krusch/Wilhelm Levison, MGH SS rer. Merov. 1, 1, Hannover ${ }^{2} 1951$, ND München 1992) 370f.

581 Cor. 14, 4. Siehe dazu Hildegard Scherer, Charismen in Korinth - das Konzept des Paulus, in: Das Charisma. Funktionen und symbolische Repräsentationen, ed. Pavlína Rychterová/Stefan Seit/Rafaela Veit (Beiträge zu den Historischen Kulturwissenschaften 2, Berlin 2008) 59-72.

${ }^{59}$ Hieronymus, Vita Hilarionis 13 (ed. Antonius Bastiaensen/Johannes Smit, Vita di Ilarione, Vite dei santi del III al VI secolo 4, Roma 1975) 70-143, hier 102-104.

${ }^{60}$ Gregorius I. papa, Dialogorum libri IV de miraculis patrum italicorum IV, 27 (ed. Adalbert de Vogüé, Grégoire le Grand, Dialogues, 1-3, Sources Chrétiennes 260, Paris 1979) vol. 3, 94. 
gedient, die Wahrhaftigkeit der Jenseitsvision eines Dieners zu unterstreichen; doch war beides nicht sein Verdienst. Die Spannung zwischen göttlicher Gabe und dämonischer Besessenheit bleibt bei Gregor unaufgelöst. Im Gegensatz dazu ist mangelnde Sprachkenntnis nicht unbedingt ein Hindernis bei der charismatischen Verkündigung des Gotteswortes. Das betont etwas Theodoret im Syrien des 5 . Jahrhunderts. Er berichtet immer wieder von Asketen, die nur Syrisch, aber kein Griechisch beherrschten; dennoch lagen einem von ihnen gleich mehrere kaiserliche Prinzessinnen zu Füßen. Sogar ein Perser, der nur einige Brocken Griechisch beherrschte, fand bei seinen Predigten in Syrien ein begeistertes Publikum. ${ }^{61}$ Nicht die perfekte Sprachbeherrschung war wichtig; der Heilige Geist musste aus der Botschaft sprechen.

\section{VERSTÄNDIGUNGSPROBLEME UND DISTANZBEWUSSTSEIN}

Auch der frühmittelalterliche Westen war von Mehrsprachigkeit geprägt. Viele Barbaren sprachen Latein, manche hatten es auch sehr gut gelernt und darin höhere Bildung erworben. Umgekehrt ironisiert Sidonius Apollinaris den burgundisch-sprachigen Römer, vor dem die Burgunder fürchteten, in ihrer eigenen Sprache Fehler zu machen. ${ }^{62}$ Die pragmatische Kommunikation, etwa in den Heeren, scheint in der Regel funktioniert zu haben; auch Dolmetscher sind immer wieder belegt. Zu alledem finden sich im vorliegenden Band zahlreiche Beispiele. Dass jeder sich in der eigenen Sprache leichter tat, wussten aber gerade diejenigen, denen die bestmögliche Vermittlung christlicher Inhalte am Herzen lag: Dulcius ab unoquoque suscipitur quod patrio sermone narratur, schreibt etwa Cassiodor. ${ }^{63}$

Pragmatische Sprachprobleme werden gelegentlich berichtet, aber meistens nicht als Konfliktursache oder als Anzeichen der Minderwertigkeit herausgearbeitet. ${ }^{64}$ So schließt Ian Wood in seinem Beitrag in diesem Band aus seinen Beobachtungen zu Gregor von Tours über die Verhältnisse in der Merowingerzeit: „Linguistic difference, however strong it was, rarely seems to have attracted attention”. Der Südgallier Eligius von Noyon zum Beispiel hatte im 7. Jahrhundert in seiner nordgallischen Diözese größte Schwierigkeiten, sich mit seinen moralischen Ermahnungen durchzusetzen; er wurde als ,Römer' beschimpft, der sich nicht in lokale Bräuche einmischen sollte; aber die Sprache wird hier nicht als Problem akzentuiert, obwohl viele moderne Autoren darüber spekuliert haben, dass er vielleicht gar nicht Fränkisch sprach. ${ }^{65}$ Im Gotenkrieg, so berichtet Prokop, hätten Totilas Leute einen byzantinischen Feldherrn armenischer Herkunft aufgegriffen, der nicht einmal Griechisch konnte und immer nur seinen Titel strategos wiederholte; da sie nichts mit ihm anfangen konnten, beschlossen sie ihn umzubringen. Die Geschichte enthält wohl eine subtile Kritik Prokops an der heterogenen Zusammensetzung der Armeen Justinians, aber keine explizit negativen Aussagen über den sprachunkundigen armenischen Offizier. ${ }^{66}$

Auf den Britischen Inseln ist häufiger von Verständigungsproblemen die Rede, wie der Beitrag von Anton Scharer in diesem Band zeigt. König Cenwalh von Wessex war wenig glücklich mit dem aus Irland gekommenen Franken Agilbert, den er zum Bischof gemacht hatte; pertaesus barbare loquelae, der ,barbarischen' Sprache überdrüssig, setzte er schließlich einen zweiten Bischof in Wessex ein. ${ }^{67}$ Das Fränkische (oder geht es hier um schlechtes Angelsächsisch?) ist hier für den Angel-

${ }^{61}$ Siehe die Vita des Aphrahat in: Theodoret, Historia ecclesiastica 8, 2 (ed. Migne, PG 82) 881-1280, hier 920; siehe Theresa Urbainczyk, Theodoret of Cyrrhus, the Bishop and the Holy Man (Michigan 2002) 77.

62 Sidonius Apollinaris, Epistolae V, 5, II (ed. Christian Luetjohann, MGH AA 8, Berlin 1887) 80f.; siehe auch Wolfgang Haubrichs, Ein namhaftes Volk - Burgundische Namen und Sprache des 5. und 6. Jahrhunderts, in: Die Burgunder. Ethnogenese und Assimilation eines Volkes, ed. Volker Gallée (Worms 2008) 135-184, hier $165 f$.

${ }_{63}$ Cassiodor, Institutiones I, Praefatio, 4 (ed. Roger A.B. Mynors, Oxford 1937) 1108 A.

${ }^{64}$ Siehe den Beitrag von Ian Wood in diesem Band.

${ }^{65}$ Vita S. Eligii (ed. Bruno Krusch, MGH SS Merov. 4, Hannover 1902) 663-741. Michel Banniard, Latin et communication orale en Gaule franque: le témoignage de la ,Vita Eligii‘, in: The Seventh Century - Changes and Continuity, ed. Jean Fontaine/Jocelyn Hillgarth (London 1992) 62-67; vgl. auch Georg Scheibelreiter, Ein Gallorömer in Flandern: Eligius von Noyon, in: Die Suche nach den Ursprüngen. Von der Bedeutung des frühen Mittelalters, ed. Walter Pohl (Forschungen zur Geschichte des Mittelalters 8, Wien 2004) 117-128, bes. 125.

${ }^{66}$ Prokop, De Bello Gothico VII, 26, 24-27 (ed. Otto Veh, München 1969) 614f.; Walter Pohl, Social language, identities and the control of discourse, in: East and West: Modes of Communication, ed. Evangelos Chrysos/Ian Wood (The Transformation of the Roman World 5, Leiden 1999) 127-141, bes. 128-32.

${ }^{67}$ Beda III, 7, ed. Spitzbart 221. Dazu und zum Folgenden siehe den Beitrag von Anton Scharer in diesem Band. 
sachsen das Barbarische und wird nicht ausreichend verstanden (oder gibt zumindest einen brauchbaren Vorwand ab, einen unliebsamen Bischof kaltzustellen). Positiver schildert Beda die Verständigungsschwierigkeiten Aidans als Bischof von Lindisfarne: „Dort ereignete es sich oft in einem sehr schönen Schauspiel, dass bei der Predigt des Bischofs, der die Sprache der Angeln nicht vollkommen beherrschte, der König selbst seinen Ealdormen und Thanen als Dolmetscher des himmlischen Wortes diente, weil der König natürlich in der langen Zeit seines Exils die Sprache der Iren schon vollständig gelernt hatte. ${ }^{\text {"68 }}$ Die Geschichte dient dazu, den König als frommen Prediger zu stilisieren, enthält aber wohl auch seine subtile Distanzierung von den irischen Missionaren. Auf der Synode von Whitby musste der erwähnte Agilbert seinen Schüler Wilfrid für sich sprechen lassen: „Er kann besser und deutlicher in der Sprache der Angeln als ich durch einen Dolmetscher erklären, was wir meinen“, erklärte er. ${ }^{69}$ Beda achtete aufmerksamer als viele kontinentale Autoren auf sprachliche Unterschiede; das entsprach sicherlich der Vielfalt sehr unterschiedlicher Sprachen auf den Britischen Inseln, lag aber wohl vor allem an der Bedeutung von fremdsprachigen Missionaren. Mehrsprachigkeit ebenso wie sprachliches Distanzbewusstsein waren während des Mittelalters auf den Britischen Inseln immer wieder ausgeprägt. Ein besonders markantes Beispiel ist der polyglotte Gerald ,of Wales' (1146-1223), der während seines langen Lebens je nach der jeweiligen Konstellation normannische, walisische, aber auch französische und englische Identifikationen in seinen Schriften in den Vordergrund rückte. ${ }^{70}$

Sicherlich war die fremde Sprache gelegentlich Anknüpfungspunkt für Vorurteile und Abgrenzungen. Der griechische Barbarentopos war zunächst sprachlich konstruiert. Abwertende Fremdwahrnehmungen und Unverständnis dafür, dass jemand die lateinische Sprache nicht beherrschte, finden sich auch in der Spätantike (etwa die berühmten Auslassungen des Sidonius Apollinaris über die Burgunder, die seine Versfüße ins Stolpern brachten). ${ }^{71}$ Notker berichtet im späten 9. Jahrhundert von einem legendären Veteran aus den Awarenkriegen, der gleich mehrere Feinde, quid nescio murmurantes, auf seiner Lanze aufspießte. ${ }^{72}$ In vielen Situationen konnten sprachliche Unterschiede, von dialektalen Nuancen bis hin zu völliger Kommunikationsunfähigkeit, den Abgrenzungen zwischen regionalen, ethnischen oder politischen Gemeinschaften Evidenz verleihen. Damit konnten zugleich Identifikationen (mit Gleichsprachigen) erleichert werden. Doch waren solche Grenzen immer punktuell und oft auch situationell. Alltägliche sprachliche Distanzerlebnisse entsprachen im Frühmittelalter offenbar noch keiner ausgebildeten Wahrnehmung fester sprachlicher und ethnischer Einheiten. ${ }^{73}$

Dazu war schon die Terminologie zu widersprüchlich: Latein war die Sprache der Romani, während die Rhomaioi Griechisch sprachen, weshalb sie im Westen Graeci genannt wurden, obwohl ihre traditionelle Selbstbezeichnung Hellenen lautete. Für Lateinisch Sprechende gab es dagegen die aus dem Germanischen stammende Fremdbezeichnung walhoz, Walaha, Walchen, Welsche, Waliser, Wallonen, die aber auch auf die Briten angewandt wurde. Theodisk/lingua theotisca war zunächst eben nicht die Sprache eines bestimmten Volkes, sondern die des Volkes, von England bis Süditalien; ${ }^{74}$ denn der Germanenbegriff des Frühmittelalters wurde selten, und dann meist territorial gebraucht, weshalb die Sprache auch nicht ,germanisch ${ }^{6}$ heißen konnte. ${ }^{75}$ Die Beispiele ließen sich fortsetzen.

${ }^{68}$ Beda III, 3, ed. Spitzbart 215.

${ }^{69}$ Beda III, 25, ed. Spitzbart 287. Siehe Paul Fouracre, Agilbert, in: The Blackwell Encyclopedia of Anglo-Saxon England, ed. Michael Lapidge/John Blair/Simon Keynes/Donald Scragg (Oxford 1999) 21.

${ }^{70}$ Robert Bartlett, Gerald of Wales. A Voice of the Middle Ages (Stroud 22006), bes. 16-30.

${ }^{71}$ Sidonius Apollinaris, Epistulae 1, 11, 3, ed. Luetjohann 16f.; cf. Jill Harries, Apollinaris and the Fall of Rome, 93 f.; Herwig Wolfram, Das Reich und die Germanen - zwischen Antike und Mittelalter (Berlin 21992) 363; Guy Halsall, Humour, History and Politics in Late Antiquity and the Early Middle Ages (Cambridge 2002) 94. Zur Sprache der Fremdwahrnehungen siehe auch den Beitrag von Fritz Lošek in diesem Band.

${ }^{72}$ Notker, Gesta Karoli II, 12 (ed. Hans F. Haefele, MGH SS rer. Germ. in us. schol., NS 12, Berlin ${ }^{2} 1980$ ) 75; cf. Walter Pohl, Die Awaren. Ein Steppenvolk in Mitteleuropa, 567-822 n. Chr. (München ${ }^{2} 2002$ ) 328. Siehe auch die Beiträge von Wolfgang Haubrichs und Hans-Werner Goetz in diesem Band, mit weiteren Beispielen.

73 Siehe dazu auch den Beitrag von Patrick Geary in diesem Band.

${ }^{74}$ Siehe die Beiträge von Herwig Wolfram, Giuseppe Albertoni und Daniela Fruscione in diesem Band, sowie Wolfram, Gotische Studien 245f.

75 Walter Pohl, Der Germanenbegriff vom 3. bis 8. Jahrhundert - Identifikationen und Abgrenzungen, in: Zur Geschichte der Gleichung ,germanisch - deutsch', ed. Heinrich Beck/Dieter Geuenich/Heiko Steuer/Dietrich Hakelberg (RGA, 
Auffällig ist auch, dass sich zwar in den Quellen immer wieder Hinweise auf eorum, nostra lingua o.ä. finden, diese Sprache aber selten eine geläufige ethnische Bezeichnung bekommt.

Wahrscheinlich liegt der relative Quellenmangel zur Identitätswirksamkeit sprachlicher Unterschiede auch daran, dass die lateinische Staats- und Kirchensprache, in einem komplexen Spannungsverhältnis mit den sich entwickelnden romanischen Volkssprachen, noch lange einen wesentlichen Rahmen politischer Identitätsbildung bot. ${ }^{76}$ Die Einzigartigkeit der katholischen gens Francorum war in lateinischen Texten festgeschrieben, etwa im ,langen Prolog ${ }^{6}$ der karolingischen Fassung der Lex Salica, in dem es hieß, dass die Franken nun die Märtyrer verehrten, die von den Römern ermordet worden waren. ${ }^{77}$ Das Latein behielt das ganze Mittelalter hindurch und darüber hinaus eine identitätsstiftende Rolle; bis hin zu jenen artifiziellen und elitären ,hisperischen Wörtern', die Kurt Smolak in seinem Beitrag als Identitätsmerkmal einer Gruppe gebildeter Iren oder Waliser beschreibt. Alle wesentlichen Zeugnisse des beginnenden Selbstbewusstseins der neuen Völker und Regna waren auf Latein abgefasst; in der Volkssprache sind, seit der gotischen Bibel, zunächst vor allem christliche Texte erhalten. Auch die volkssprachlichen Epen, die aus späteren Jahrhunderten überliefert sind, waren offenbar nicht vorrangig Medien der ethnischen Identitätsbildung; das bedeutendste angelsächsische Heldenlied (Beowulf) handelt bekanntlich von einem Dänen, das erfolgreichste kontinentale Epos (das Nibelungenlied) von den untergegangenen Völkern der Burgunder und Hunnen. ${ }^{78}$ Vieles deutet darauf hin, dass der Kommunikationsraum, in dem über Zugehörigkeit und Fremdartigkeit verhandelt wurde, in den ersten Jahrhunderten des Mittelalters eben nicht aus einigermaßen abgrenzbaren sprachlichen Einheiten bestanden hat, in denen sich über kulturelle Besonderheit ein Bewusstsein einer übersichtlichen ethnisch-linguistisch gegliederten Landschaft entwickeln konnte. Westgoten, Langobarden und Franken konnten über die Jahrhunderte ihre Sprache schrittweise aufgeben, weil sie in diesem Fall eben kein unverzichtbares Zugehörigkeitskriterium war.

Das muß nicht heißen, dass im Frühmittelalter - etwa zwischen Sprechern romanischer oder germanischer Sprachen - nicht mehr oder weniger Distanzbewusstsein, Vorurteile, Ablehnung bestanden haben mögen, wie sie seit dem 9. Jahrhundert ja zunehmend belegt sind.$^{79}$ Sie wurden nur kaum in schriftlicher Form aktualisiert und in politische Diskurse umgesetzt. Denn der lateinisch-christliche Diskurs gab den Völkern und Sprachen vor allem andere Bedeutungen. Zwar stand zur Abgrenzung der vorurteilsgesättigte Barbaren/Heiden-Begriff zur Verfügung; gentes barbaricae waren ja gerade die heidnischen Völker, die keine der heiligen bzw. Kultursprachen beherrschten. Doch unter der Herrschaft christianisierter ,barbarischer' Gentes wurde gerade diese ideologische Unterscheidung bald problematisch. In der Karolingerzeit wurde noch einmal die Scheidung zwischen dem wiedererrichteten christlichen Imperium und den heidnischen-barbarischen Gentes eingeschärft. Doch sie wurde zugleich relativiert durch den universellen Missionsauftrag, denn alle Gentes sollten ja die Heilsbotschaft vernehmen. Dabei konnten Sprachdifferenzen durchaus pragmatische Wichtigkeit erlangen, wie ein Brief des Mönches Blancidius, eines Schülers Alcuins, beweist. Er befand sich „in den Schlupfwinkeln an der Donau, in den Bergen der Slawen und im dichten Tannenwald", wo er „wie ein Frosch im Sumpf saß, die Sprache nicht verstand und statt Feige Eichel riss ...".80 Die Mission zwang zu pragmatischem Umgang mit Sprachunterschieden, und viele waren dieser Herausforderung nicht gewachsen. Doch sonst bewegten sich die Alltagserfahrungen von Kommunikationsschwierigkeiten mit Fremd(sprachig)en auf einer anderen Ebene, für die herrschende Diskurse keine durchgängige Deutung und Legitimierung boten.

Die Erfahrung der Fremdheit nämlich hat ein gefährliches Potential: Wenn sie auf eine einleuchtende Erklärung, ein plausibles Bedrohungsbild trifft, kann sie schnell zum Konflikt führen. Sie ist

Erg. Bd. 34, Berlin/New York 2004) 163-183. Siehe auch die Beiträge von Daniela Fruscione und Giuseppe Albertoni in diesem Band.

${ }^{76}$ Siehe auch die Beiträge von Michel Banniard, Roger Wright, Kurt Smolak und Michael Richter in diesem Band.

${ }^{77}$ Lex Salica, Prologus (ed. Karl August Eckhardt, MGH LL nat. Germ. 4, 2, Hannover 1969) 8.

${ }^{78}$ Vgl. den Beitrag von Uta Goerlitz in diesem Band.

79 Weitere Beispiele finden sich bei Paul Meyvaert, Rainaldus est malus scriptor Francigenus - voicing national antipathy in the Middle Ages, in: Speculum 66 (1991) 743-763.

${ }^{80}$ Appendix ad Alcuini epistulas 2 (ed. Ernst Dümmler, MGH Epp. IV, EE Karolini aevi II, Berlin 1895) 484. Siehe Herwig Wolfram, Salzburg, Bayern, Österreich. Die Conversio Bagoariorum et Carantanorum und die Quellen ihrer Zeit (Wein/München 1995) 72. 
daher besonders anfällig für politischen Missbrauch. Das ist unter verschiedenen Umständen sehr unterschiedlich und fügt sich auch nicht in eine lineare Entwicklung, etwa hin zur modernen Nation. Im ,dunklen' Frühmittelalter wurden Sprachdifferenzen, ebenso wie ethnische Vielfalt, offenbar seltener als Problem wahrgenommen und politisch benützt als in der aufgeklärten Moderne. Doch lässt sich dabei noch nach Zeit und Ort differenzieren. Unsere Aufgabe ist es daher nicht, die historischen Unterschiede einzuebnen, um ein ,allgemein-menschliches' Bild von der Wichtigkeit der Sprache für die Selbst- und Fremdbilder ethnischer Gruppen zu gewinnen. Wir wollen wissen, wann, wie und in welchem Kontext die Rede von den sprachlichen Unterschieden Bedeutung gewann und wozu sie dienen sollte. Dazu kann dieser Band wesentliche neue Erkenntnisse beitragen. Eine gemeinsame Sprache erleichterte sicherlich die Identifikation mit einer ethnischen Gruppe; und doch kamen bei der Neugestaltung der ethnischen und politischen Landschaft nach dem Zerfall des Römischen Reiches sprachliche, ethnische und politische Identitäten keineswegs zur Deckung. Sprache erschloss vielerlei Gemeinschaften und erlaubte ganz unterschiedliche Abgrenzungen; ein privilegierter oder gar ausschließlicher Bezug zu ethnischer Identität ist in den frühmittelalterlichen Quellen nicht erkennbar. 